\title{
A phase II trial of the effect of perindopril on hand-foot skin reaction (HFSR) incidence and severity in patients receiving regorafenib for refractory $\mathrm{mCRC}$
}

\author{
Barbara L. Melosky ${ }^{1}$. Howard John Lim ${ }^{1}$. Janine Marie Davies ${ }^{1}$. Sharlene Gill ${ }^{1}$. Christian K. Kollmannsberger ${ }^{1}$. \\ Maria Yi Ho ${ }^{1}$. Solomon A. Vandt ${ }^{1}$. Daniel John Renouf ${ }^{1}$
}

Received: 19 October 2018 / Accepted: 26 November 2018 / Published online: 8 December 2018

(c) The Author(s) 2018

\begin{abstract}
Purpose Regorafenib is an oral multi-kinase inhibitor that offers an OS benefit to patients with mCRC refractory to standard therapy (Grothey et al., in Lancet 381:303-312, 2013), but comes with potential significant toxicities including grade 3 hand-foot skin reaction (HFSR). The pathogenesis of regorafenib-induced HFSR is not well established, but may be related to alterations in the capillary endothelium. We hypothesized that perindopril, an angiotensin-converting enzyme (ACE) inhibitor, indicated for the treatment of hypertension (Ceconi et al., in Cardiovasc Res 73:237-246, 2007), and which plays a role in preventing endothelial dysfunction, may help to prevent or reduce the severity of regorafenib-induced HFSR.

Patients and methods In this single-center phase II open-label trial, patients with refractory mCRC were treated with both regorafenib (160 mg/day) and perindopril (4 mg/day) for 21 days per 28-day cycle. The primary end point was to assess the proportion of patients with any grade HFSR toxicity. Secondary end points included time to development of worst (grade 3) HFSR, reduction of all grades of hypertension and all grade toxicities, as well as progression-free survival. All toxicities were evaluated using CTCAE v4.03.

Results A planned interim analysis was performed after ten evaluable patients had completed their first cycle of study treatment. As 50\% (5/10) experienced grade 3 HFSR, enrolment was stopped as the addition of perindopril did not lead to a reduced level of HFSR compared with regorafenib alone. Other grade 3 toxicities included hypertension (16.7\%) and increased AST (16.7\%).

Conclusion The addition of an ACE inhibitor perindopril to regorafenib did not reduce HFSR incidence or severity in patients with refractory mCRC.
\end{abstract}

Keywords Regorafenib $\cdot$ Colorectal cancer $\cdot$ Perindopril $\cdot$ ACE inhibitor $\cdot$ HFSR

\section{Abbreviations}

AE Adverse event

ALT Alanine aminotransferase

AST Aspartate aminotransferase

BCCA BC Cancer Agency

BSC Best supportive care

CBC Complete blood count

CRC Colorectal cancer

This study was supported through a grant from Bayer Canada.

These results were previously presented at ASCO 2018.

Barbara L. Melosky

BMelosky@bccancer.bc.ca

1 BC Cancer Vancouver Centre, 600-10th Ave W, Vancouver, BC V5Z 4E6, Canada
CTC(AE) Common Terminology Criteria (for AEs)

ECOG Eastern Cooperative Oncology Group

EGFR Epidermal growth factor receptor

FGFR Fibroblast growth factor receptor

FU 5-Fluorouracil

HR Hazard ratio

HFSR Hand-foot skin reaction

KM Kaplan-Meier

mCRC Metastatic colorectal cancer

OS Overall survival

PARRICA Perindopril and regorafenib in mCRC

PDGF Platelet-derived growth factor

PDGFR Platelet-derived growth factor receptor

PFS Progression-free survival

PS Performance status 


$\begin{array}{ll}\text { RECIST } & \begin{array}{l}\text { Response Evaluation Criteria in Solid } \\ \text { Tumors }\end{array} \\ \text { VEGF } & \text { Vascular endothelial growth factor } \\ \text { VEGFR } & \text { Vascular endothelial growth factor receptor } \\ \text { vs } & \text { Versus }\end{array}$

\section{Purpose}

Colorectal cancer is the second most frequently diagnosed cancer in males and the third most frequently diagnosed cancer in females [1]. The disease remains the second most frequent cause of cancer death in males and the third most frequent cause of cancer death in females [1].

The median overall survival (OS) for patients with unresectable mCRC who receive only best supportive care (BSC) is 5-6 months [2]. Palliative treatment with systemic chemotherapy prolongs survival and maintains quality of life [3].

For many years, 5-fluorouracil (5-FU) was the only treatment, but the approval of irinotecan, oxaliplatin, fluoropyrimidines, as well as monoclonal antibodies such as bevacizumab targeting VEGF and cetuximab and panitumumab targeting EGFR growth factors, led to the development of additional regimens. The ideal combination and sequence of the different agents are still being determined.

Regorafenib has shown efficacy in mCRC patients who have been previously treated with fluoropyrimidine-based chemotherapy, oxaliplatin, irinotecan, an anti-VEGF therapy, and, if $R A S$ wild type, an anti-EGFR therapy $[4,5]$. It is a multi-kinase inhibitor belonging to a unique class of orally administered small molecule therapeutics targeting multiple protein kinases, including kinases involved in tumour angiogenesis (VEGFR1, -2, -3, TIE2), oncogenesis (KIT, RET, RAF-1, BRAF, BRAFV600E), and the tumour microenvironment (PDGFR, FGFR) [6]. The phase III CORRECT trial demonstrated regorafenib's efficacy in mCRC patients who were refractory to standard therapy, in which regorafenib prolonged OS compared with placebo [4]. The efficacy and safety results were confirmed in the randomised CONCUR trial [5]. The study met its primary OS end point, 8.8 months for regorafenib as compared to 6.3 months with placebo [HR $0.550,95 \%$ CI (0.395-0.765), $P=0.0002$ (1-sided)].

Hand-foot skin reaction (HFSR) is the most significant $\mathrm{AE}$ experienced by regorafenib-treated patients. Efforts are needed to reduce HFSR related to regorafenib, as it causes a significant decrease in a patient's quality of life, requires dose reductions or modifications, and can ultimately lead to treatment discontinuation. In the CORRECT and CONCUR trials, $47-74 \%$ of patients treated with regorafenib experienced any grade of HFSR, with about $17 \%$ experiencing grade 3 HFSR $[4,5]$. Skin toxicity was also the most frequent adverse event necessitating dose modification or interruption.
The pathogenesis of regorafenib-induced HFSR has not yet been fully established; however, the capillary endothelium may be the first target in HFSR. Abnormal signalling interrupting the VEGF and PDGF receptors may lead to alteration of small vessels [7], which are traumatized by frequent impacts or pressure requiring endothelial repair [8]. An inflammatory response has also been hypothesized [8].

Hypertension is also an issue with regorafenib, affecting up to $28 \%$ of patients in the CONCUR and CORRECT trials, with 12 and $7 \%$ reporting severe hypertension, respectively $[4,5]$.

Perindopril is an angiotensin-converting enzyme (ACE) inhibitor indicated for the treatment of hypertension, congestive heart failure, and for the treatment of hypertensive and/ or post-MI patients with stable coronary artery disease [9]. ACE inhibitors protect the vascular endothelium by decreasing the concentration of angiotensin II, which inhibits nitrous oxide (NO) production and activity while increasing the concentrations of bradykinin, a vasodilator and stimulator of NO [10]. Different ACE inhibitors have demonstrated differing abilities to improve vascular endothelial function as demonstrated through flow-mediated vasodilation [11], with perindopril being most effective [10]. As a vasodilator, perindopril may be beneficial in preventing or reducing the severity of regorafenib-induced HFSR, possibly by helping to restore the normal balance between angiotensin II and bradykinin, and reducing inflammation (TNF-a) to reverse endothelial dysfunction [10, 12-14].

The hypothesis underlying this trial was that the coadministration of perindopril with regorafenib would mitigate HFSR symptoms, increasing the treatment duration.

\section{Methods}

\section{Study design and participants}

This phase II Study of Perindopril and Regorafenib in Metastatic Colorectal Cancer (PARICCA) was an open label, single arm trial of patients with refractory mCRC, conducted to measure the incidence and severity of HFSR and hypertension using the CTCAE v4.03 criteria, in patients receiving both regorafenib (160 mg/day) and perindopril (4 mg/day).

Eligible patients had a pathologic documentation of stage IV adenocarcinoma of the colon or rectum and progressed on/after all approved drugs for mCRC including FU-based chemotherapy, oxaliplatin, irinotecan, anti-VEGF therapy, and, if $R A S$ wild type, an anti-EGFR therapy. Patients had to have refractory or progressive disease within 3 months following the last administration of approved standard therapies, or experienced intolerance to previous therapy. Patients treated with oxaliplatin in the adjuvant setting had to progress during or within 6 months of completion of adjuvant 
therapy. Patients were aged 18 years or older; had an Eastern Cooperative Oncology Group (ECOG) performance status of 0 or 1 within 14 days prior to the initiation of study treatment; life expectancy of at least 3 months; and adequate bone marrow, liver and renal function at the start of the trial. Patients were not eligible if they had previously received regorafenib; had an uncontrolled medical disorder deemed significant by the treating physician; and history of hereditary/idiopathic angioedema, or angioedema related to previous treatment with an ACE inhibitor.

The study was conducted at the BC Cancer Vancouver Center. The University of British Columbia Research Ethics Board approved the protocol, ClinicalTrials.gov Identifier NCT02651415. The trial followed the guiding principles of the Declaration of Helsinki and good clinical practice and complied with all local laws and regulations. Participants provided written informed consent before enrolment; when a patient was not capable of providing a signature, an oral statement of consent could be provided in the presence of a witness.

\section{Study end points}

The primary end point was the incidence of all grade toxicities for HFSR defined by CTCAE v4.03 criteria. The incidence of HFSR was expressed as the number of patients experiencing any grade HFSR. The primary end point was a 50\% reduction in all grades of HFSR based on CTCAE v 4.03 criteria (i.e. from $47 \%$ any grade HFSR to $24 \%$ of patients).

Secondary end points were the incidence of all grade hypertension and toxicity; maximal severity of HFSR; time to development of stage $3 \mathrm{HFSR}$; and progression-free survival (PFS). All grades of hypertension were evaluated using CTCAE v4.03, measured weekly for the first 6 weeks while patients were on the study drug, then every second week during treatment with perindopril and during the 30-day follow-up period (post-therapy).

All grades of AE (including HFSR) were evaluated using CTCAE v4.03 at baseline and day 1 of each cycle while they were on the study drug and during the 30-day follow-up period (post-therapy). PFS was evaluated based on RECIST v1.1 criterion, with $20 \%$ progression of existing metastases or any new metastases.

Information on previous experience with HFS was collected as per patient recollection only at enrolment (no chart review). This was an exploratory analysis to determine if previous HFS influences the rate and severity of HFSR in this study.

Patients were followed for survival. For subjects who discontinued study treatment and did not experience PD, available tumour assessments were recorded in the CRF until documented PD.

\section{Procedures}

The dose and schedule of regorafenib used in this trial was the approved dose of $160 \mathrm{mg}$ per day from data accumulated in previous regorafenib phase III trials $[4,5]$. Perindopril erbumine $4 \mathrm{mg}$ was administered daily for 21 days of a 28-day cycle [9]. Perindopril was administered in the morning on an empty stomach. Regorafenib was administered $160 \mathrm{mg}$ daily for 21 days of a 28-day cycle, with a low fat breakfast $1 \mathrm{~h}$ after perindopril. All patients received BSC.

Predefined dose modifications were permitted to manage clinically significant treatment-related AEs. Patients who required dose reductions could re-escalate the dose up to $160 \mathrm{mg}$ daily at the discretion of the investigator once the AE resolved to baseline levels. Treatment was discontinued permanently if the AE did not recover after a 4-week interruption or after dose reduction by two dose levels.

Patients were treated until clinical radiological disease progression based on RECIST v1.1 criterion, death, unacceptable toxicity, withdrawal of consent by the patient, decision by the treating physician that discontinuation would be in the patient's best interest, or substantial non-compliance with the protocol. If in the investigator's opinion treatment with regorafenib provided clinical benefit to a patient experiencing disease progression, the patient could continue treatment.

Patients were seen by a physician every week for the first cycle, every 2 weeks for the second cycle, at the start of each subsequent cycle, at the end of treatment, and every month after cessation of treatment until death or data cutoff. ECOG status and concomitant medications were assessed at the start of treatment and at each physician visit. Blood pressure and LFTs were done weekly for the first cycle, on days 1 and 7 in the second cycle, on day 1 of each subsequent cycle, and at the follow-up visit. Lipase, electrolytes, TSH, and chemistry were collected on day 1 of each cycle. CEA, $\mathrm{CBC}$, and creatinine were evaluated on day 1 of each cycle and at the end of treatment. A 12-lead ECG was administered on day 11 of each cycle and at the end of treatment. Other tests were administered as per the standard of care for regorafenib and perindopril. The study protocol mandated a patient education module that included a patient education brochure regarding the management of HFSR and preventative measures to manage this side effect.

\section{Study interim analysis and discontinuation rule}

The study was to be discontinued if five of the first ten patients exhibited a Grade 3 HFSR, or if the HFSR was more severe with the addition of perindopril than with regorafenib alone. 


\section{Statistical analysis}

Demographic and other baseline characteristics were listed and summarized. Qualitative data were summarized using frequencies and percentages; quantitative data were summarized using descriptive statistics. Secondary safety variables were summarized using descriptive statistics and exploratory graphical presentations of the data. Statistical analyses were performed using SAS 9.3.

The primary analysis set consisted of all evaluable patients. An evaluable patient was defined as an eligible patient who received at least one cycle of study medication. The safety set consisted of all patients who received at least one dose of study medication. RECIST criteria were used by the investigator to determine PFS and OS end points.

\section{Sample size assumptions}

A $10 \%$ reduction in all grades of HFSR is a clinically meaningful reduction, with an alpha level of 0.05 and a power of $80 \%$ for the sample size calculation.

\section{Results}

This study was initiated in August 2016 and the last patient visit was conducted in March 2018. Although 12 patients were accrued over a 9-month period, 2 patients withdrew in the first cycle; as such, 10 patients were evaluable.

The baseline characteristics of the patients are shown in Table 1. Equal numbers of males and females (5:5) were enrolled, and the median age of the trial participants was 60 years (range 49-72), and at the baseline screening assessment $70 \%$ had ECOG 1 performance status. Forty percent of patients had experienced previous HFS, most likely from previous therapy.

Seven patients had moderately differentiated tumours, seven had three or more previous systemic anticancer therapies for metastatic disease, and eight patients had received FOLFOX or FOLFIRI before starting on this therapy.

The median days on treatment was 51.9 days. Four patients completed three or four treatment cycles (Table 2). Half of the patients received a dose reduction in this study, with three requiring one dose reduction and two requiring two dose reductions. Nine patients had a dose delay or hold, with five experiencing a delay of $\geq 1$ week and $40 \%$ experiencing a delay of $\geq 2$ weeks. Seven discontinued treatment due to disease progression.

After ten evaluable patients had completed their first cycle of study treatment, a planned interim analysis was conducted. As five of the patients enrolled experienced grade 3 HFSR, the study was discontinued, as the addition of perindopril was unlikely to lead to a reduced level
Table 1 Baseline demographics in the evaluable patient population

\begin{tabular}{ll}
\hline Age (years) & $N=10(\%)$ \\
& $\begin{array}{c}\text { Median } 60.65 \\
\text { (range, } 49-\end{array}$ \\
Sex & $72)$ \\
Female & $5(50 \%)$ \\
Male & $5(50 \%)$ \\
Baseline ECOG & \\
0 & $3(30 \%)$ \\
1 & $7(70 \%)$ \\
Ethnicity & \\
Asian & $5(50 \%)$ \\
Black & $1(10 \%)$ \\
White & $4(40 \%)$ \\
Previous HFSR or HFS & \\
Yes & $4(40 \%)$ \\
If yes, and had HFSR during the study & $3(30 \%)$ \\
Stage at diagnosis & \\
II & $1(10 \%)$ \\
III & $4(40 \%)$ \\
IV & $5(50 \%)$ \\
Previous systemic anticancer therapies & \\
2 & $3(30 \%)$ \\
3 & $6(60 \%)$ \\
4 & $1(10 \%)$ \\
\hline & \\
\hline &
\end{tabular}

Table 2 Treatment: dose levels

\begin{tabular}{ll}
\hline Mean days of treatment $(N=10)$ & $55.9(6-118)$ days \\
Total number of cycles received & $N=10(\%)$ (evaluable) \\
1 & $2(20 \%)$ \\
2 & $4(40 \%)$ \\
3 & $2(20 \%)$ \\
4 & $2(20 \%)$ \\
Lowest dose reduction level & \\
$0(160 \mathrm{mg}$ PO OD) & $5(50 \%)$ \\
$-1(120 \mathrm{mg}$ PO OD) & $3(30 \%)$ \\
$-2(80 \mathrm{mg}$ PO OD) & $2(20 \%)$ \\
Longest dose delay/hold & \\
No delays & $1(10 \%)$ \\
$<1$ week & $0(0 \%)$ \\
$\geq 1$ week & $5(50 \%)$ \\
$\geq 2$ weeks & $4(40 \%)$ \\
\hline
\end{tabular}

of regorafenib-induced HFSR compared with regorafenib alone.

The most frequent grade 3 AEs were HFSR (50\%), hypertension (20\%), and increased AST (20\%). All other toxicities are described in Table 3. Two patients 
Table 3 Clinical adverse events and laboratory measurements of all grades and of grade 3 and above

\begin{tabular}{|c|c|c|}
\hline Any event & $N$ all grades & $N$ grade $\geq 3$ \\
\hline \multicolumn{3}{|l|}{ Clinical adverse event } \\
\hline Hand-foot skin reaction & 7 & 5 \\
\hline Fatigue & 7 & 2 \\
\hline Hypertension & 6 & 2 \\
\hline Pain, abdomen & 6 & 1 \\
\hline Anorexia & 6 & \\
\hline $\begin{array}{l}\text { Oral mucositis (dry mouth, sore throat, } \\
\text { mucosal infection) }\end{array}$ & 6 & \\
\hline Diarrhoea & 5 & \\
\hline Muscle pain (myalgia) & 5 & \\
\hline Rash or desquamation & 4 & 1 \\
\hline Nausea & 4 & \\
\hline Constipation & 4 & \\
\hline Voice changes/hoarse voice & 4 & \\
\hline Arthralgia (joint pain) & 4 & \\
\hline Pain extremity/neuralgia & 3 & 1 \\
\hline Infection (UTI) & 3 & \\
\hline Fever (pyrexia) & 3 & \\
\hline Dry skin & 3 & \\
\hline Vomiting & 3 & \\
\hline Ascites & 3 & \\
\hline Headache & 3 & \\
\hline Thigh, groin, pelvic pain & 2 & 1 \\
\hline Non-cardiac chest pain & 2 & 1 \\
\hline Back pain & 2 & \\
\hline Rectal/anal pain & 2 & \\
\hline Dysphagia (difficulty swallowing) & 2 & \\
\hline Oedema (ankle) & 2 & \\
\hline Dyspnoea (shortness of breath) & 2 & \\
\hline Dry cough & 2 & \\
\hline Pneumonia & 1 & 1 \\
\hline Cramps (leg) & 1 & \\
\hline Erythaema face & 1 & \\
\hline Haemoptysis (spitting blood) & 1 & \\
\hline Insomnia & 1 & \\
\hline Pruritis (itchy) & 1 & \\
\hline Pain, soles of feet, toe & 1 & \\
\hline Weakness & 1 & \\
\hline Chills & 1 & \\
\hline Nose bleed (epistaxis) & 1 & \\
\hline Weight loss & 1 & \\
\hline Paraesthesia & 1 & \\
\hline \multicolumn{3}{|l|}{ Laboratory values } \\
\hline $\begin{array}{l}\text { Increased AST (aspartate aminotrans- } \\
\text { ferase) }\end{array}$ & 3 & 2 \\
\hline Hypokalaemia & 2 & 1 \\
\hline Hyperbilirubinaemia & 2 & 1 \\
\hline Increased alk phosphatase & 1 & 1 \\
\hline Increased lipase & 1 & 1 \\
\hline
\end{tabular}

Table 3 (continued)

\begin{tabular}{ll}
\hline Any event & $N$ all grades $N$ grade $\geq 3$ \\
\hline Elevated INR & 1 \\
Haematuria & 1 \\
\hline
\end{tabular}

Table 4 Outcomes for evaluable patients $(n=10)$

\begin{tabular}{lc}
\hline Outcome & Months (95\% CI) \\
\hline Median PFS from study start & $2.60(1.74-3.61)$ \\
Median OS from study start (months) & $7.33(2.33-11.76)$ \\
Median OS from diagnosis of metastasis & $30.49(12.19-43.53)$ \\
(months) & \\
\hline
\end{tabular}

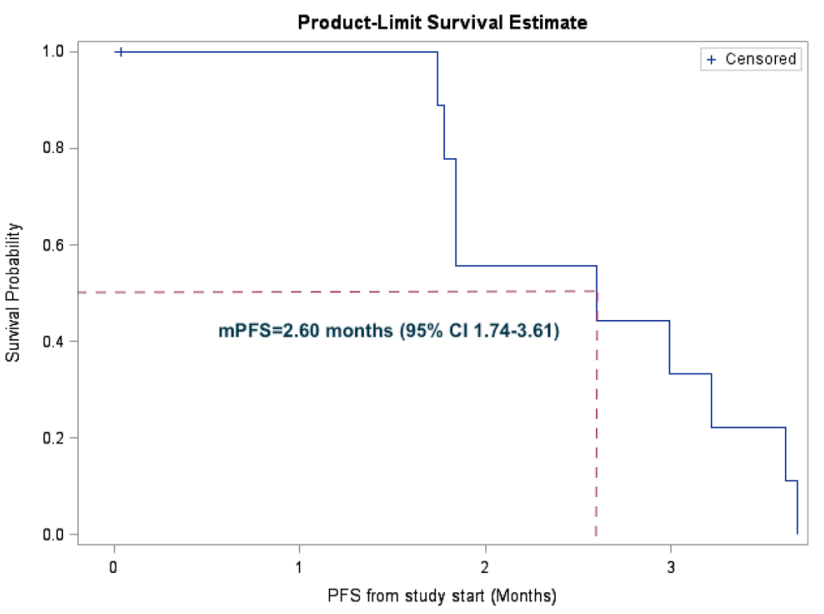

Fig. 1 Kaplan-Meier curve of progression-free survival. One patient was censored and assumed to progress 1 day after start of study (as per protocol). This patient is indicated by ' + ' at the start of the line on the plot

experienced grade 1 or 2 HFSR. Interestingly, three of the four patients who had previous HFS experienced grade 3 HFSR while on this study.

The median days to HFSR resolution by one grade was 13 days (range 2-109 days) for all transitions $(n=17), 5.5$ days (range 2-25) for grade 2 to grade $1(n=6)$, and 25 days (range 15-109 days) for grade 1-0 $(n=6)$. The number of days to complete HFSR resolutions from grade 3 to 0 was 51 days (range $36-125$ days) $(n=5)$.

Patient outcomes are shown in Table 4. PFS from study start was 2.60 months (95\% CI 1.74-3.61). The median OS was 7.33 months (95\% CI 2.33-11.76). PFS and OS are shown in Fig. 1a, b, respectively. 


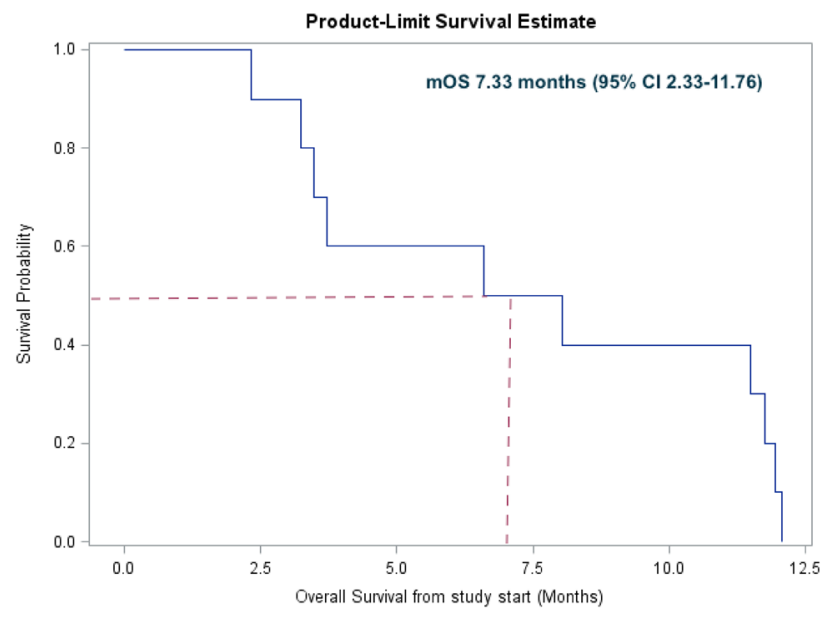

Fig. 2 Kaplan-Meier curve of overall survival

\section{Discussion}

Regorafenib has demonstrated OS benefits for patients with mCRC refractory to standard therapies. Regorafenib-induced HFSR can significantly impact the quality of life, requiring dose reductions or modifications. Our study objective was to determine if perindopril had any effect on the incidence of regorafenib-induced HFSR in refractory mCRC. Our hypothesis was that the vasodilation of small vessels with perindopril would mitigate regorafenib-associated HFSR and hypertension in an mCRC context. Although there were many different ACE inhibitors to consider, we chose perindopril as it is least likely to cause hypotension. The results demonstrated that the addition of perindopril to regorafenib did not reduce the rates of HFSR, and the trial was discontinued at the interim analysis due to high rates of grade 3 HFSR (Fig. 2).

Although the study numbers were small, the rate of grade 3 HFSR was much higher than expected based on a previous trial. In the CORRECT and CONCUR trials, only $17 \%$ and $16.2 \%$ of patients experienced grade $3 \operatorname{HFSR}[4,5]$.

An interesting observation from this small study was that patients with a previous (self-reported) history of HFS secondary to capecitabine had a high rate of regorafenibinduced HFSR. The duration of time to resolution of HFSR was long in this trial, especially for grade 3 HFSR to resolve completely (51 days). Previous HFS and duration of time to resolution may be considered in future regorafenib studies.

Efforts need to be made to find new dosing strategies and therapeutic combinations to reduce HFSR. One promising strategy is the Regorafenib Dose Optimization Study (ReDOS) [15]. This randomized phase II study examined initiating regorafenib at low doses $(80 \mathrm{mg})$ and then escalating to $160 \mathrm{mg}$, with the end point of increasing the proportion of patients who completed two cycles of treatment and initiated a third, compared to patients who started on the standard dose. The initial results indicated that the study met its primary end point with $43 \%$ of the patients in the dose escalation cohort initiating a third dose versus $25 \%$ of patients on standard therapy. Also, median OS, PFS and overall rates of grade 3/4 toxicity were more favourable in the dose escalation arm. This study also tested the use of a steroid cream, clobetasol propionate, to reduce HFSR, but the results for this are yet to be reported.

Further studies to reduce HFSR for regorafenib and other multi-targeted tyrosine kinase inhibitors are needed, as these may significantly impair the optimal treatment benefit and the patient's quality of life.

Acknowledgements The authors would like to thank Chrystal Palaty for writing assistance and project management and Colleen McGahan for statistical assistance.

Funding This was an investigator-initiated trial. Bayer provided the study drug and collaborated with the investigators on protocol design and provided funds for writing assistance and statistics. Bayer did not have any role in data collection or interpretation, nor did they have any influence in the preparation of this paper. The two principal investigators (BM and DR) had final responsibility for the content of the report and for the decision to submit for publication. All authors had access to the study data and reviewed the paper.

\section{Compliance with ethical standards}

Conflict of interest Barbara L. Melosky: Advisory Board/honorarium: Bayer, Pfizer, Astra Zeneca, Boerhinger Ingleheim, Roche. Research funding: Bayer. Howard John Lim: honoraria from Bayer. Janine Davies: honoraria from Bayer. Sharlene Gill: no relevant disclosures. Christian K. Kollmannsberger: no relevant disclosures. Maria Ho: no relevant disclosures. Solomon A. Vandt: no relevant disclosures. Daniel John Renouf: Advisory board/honorarium: Celgene; Shire; Bayer. Research funding: Bayer.

Open Access This article is distributed under the terms of the Creative Commons Attribution 4.0 International License (http://creativeco mmons.org/licenses/by/4.0/), which permits unrestricted use, distribution, and reproduction in any medium, provided you give appropriate credit to the original author(s) and the source, provide a link to the Creative Commons license, and indicate if changes were made.

\section{References}

1. Canadian Cancer Statistics (2018) http://www.cancer.ca/ /media/ cancer.ca/CW/cancerinformation/cancer101/Canadian cancer stati stics/Canadian-Cancer-Statistics-2018-EN.pdf?la=en. Canadian Cancer Society, 2018. Accessed 18 June 2018

2. Scheithauer W, Rosen H, Kornek GV et al (1993) Randomised comparison of combination chemotherapy plus supportive care with supportive care alone in patients with metastatic colorectal cancer. BMJ 306:752-755

3. Venook AP, Niedzwiecki D, Lenz HJ et al (2017) Effect of firstline chemotherapy combined with cetuximab or bevacizumab on overall survival in patients with KRAS wild-type advanced or 
metastatic colorectal cancer: a randomized clinical trial. JAMA 317:2392-2401

4. Grothey A, Van Cutsem E, Sobrero A et al (2013) Regorafenib monotherapy for previously treated metastatic colorectal cancer (CORRECT): an international, multicentre, randomised, placebocontrolled, phase 3 trial. Lancet 381:303-312

5. Li J, Qin S, Xu R et al (2015) Regorafenib plus best supportive care versus placebo plus best supportive care in Asian patients with previously treated metastatic colorectal cancer (CONCUR): a randomised, double-blind, placebo-controlled, phase 3 trial. Lancet Oncol 16:619-629

6. STIVARGA ${ }^{\circledR}$ PRODUCT MONOGRAPH (2018) https://omr. bayer.ca/omr/online/stivarga-pm-en.pdf. Bayer Inc. Accessed 18 June 2018

7. Erber R, Thurnher A, Katsen AD et al (2004) Combined inhibition of VEGF and PDGF signaling enforces tumor vessel regression by interfering with pericyte-mediated endothelial cell survival mechanisms. FASEB J 18:338-340

8. Robert C, Soria JC, Spatz A et al (2005) Cutaneous side-effects of kinase inhibitors and blocking antibodies. Lancet Oncol 6:491-500

9. Perindopril Product Monograph (2017) https://www.servier.ca/ sites/default/files/webform/products/PMCOVERSYLPLUSEnglis h13Oct2017.pdf?ts $=1511532317$. Servier Canada Inc., Accessed 18 June 2018
10. Taddei S (2012) New evidence for endothelial protection. Medicographica 34:17-24

11. Anderson TJ, Elstein E, Haber $\mathrm{H}$ et al (2000) Comparative study of ACE-inhibition, angiotensin II antagonism, and calcium channel blockade on flow-mediated vasodilation in patients with coronary disease (BANFF study). J Am Coll Cardiol 35:60-66

12. Ceconi C, Fox KM, Remme WJ et al (2007) ACE inhibition with perindopril and endothelial function. Results of a substudy of the EUROPA study: Pertinent. Cardiovasc Res 73:237-246

13. Ceconi C, Francolini G, Olivares A et al (2007) Angiotensinconverting enzyme (ACE) inhibitors have different selectivity for bradykinin binding sites of human somatic ACE. Eur J Pharmacol 577:1-6

14. Zhuo JL, Mendelsohn FA, Ohishi M (2002) Perindopril alters vascular angiotensin-converting enzyme, AT(1) receptor, and nitric oxide synthase expression in patients with coronary heart disease. Hypertension 39:634-638

15. Bekaii-Saab TS, Ou F-S, Anderson DM et al (2018) Regorafenib dose optimization study (ReDOS): Randomized phase II trial to evaluate dosing strategies for regorafenib in refractory metastatic colorectal cancer (mCRC): An ACCRU Network study. J Clin Oncol 36:611-611 